\title{
Danish Register of chronic obstructive pulmonary disease
}

This article was published in the following Dove Press journal:

Clinical Epidemiology

25 October 2016

Number of times this article has been viewed

Peter Lange ${ }^{1,2}$

Sandra Søgaard Tøttenborg'

Anne Dichmann Sorknæs ${ }^{3}$

Jørgen Steen Andersen ${ }^{4}$

Mette Søgaard ${ }^{5}$

Henrik Nielsen ${ }^{5}$

Reimar Wernich Thomsen ${ }^{5}$

Katrine Abildtrup Nielsen ${ }^{6}$

'Section of Social Medicine,

Department of Public Health,

University of Copenhagen, ${ }^{2}$ Lung

Diseases Department, Respiratory

Medicine Section, Lung Diseases

Department, Hvidovre Hospital,

University of Copenhagen,

Copenhagen, ${ }^{3}$ Medical Department,

Odense Universitetshospital-

Svendborg Hospital, Svendborg,

${ }^{4}$ Danish College of General

Practitioners, Copenhagen,

${ }^{5}$ Department of Clinical Epidemiology,

Aarhus University Hospital, ${ }^{6}$ Registry

Support Centre of Clinical Quality

and Health Informatics (West), Århus,

Denmark
Correspondence: Peter Lange

Section of Social Medicine, Department of Public Health, University of Copenhagen,

PO Box 2099, Øster Farimagsgade 5,

DK-1014 Copenhagen K, Denmark

Tel +453532 7965

Fax +45 3535 । I I ।

Email peter.lange@sund.ku.dk
Aim of database: The Danish Register of Chronic Obstructive Pulmonary Disease (DrCOPD) is a nationwide database aiming to describe the quality of treatment of all patients with chronic obstructive pulmonary disease (COPD) in Denmark.

Study population: DrCOPD comprises data on all patients with a diagnosis of COPD. In the hospital setting, both in- and outpatients are included. In the setting of the general practice (GP), DrCOPD aims to include all patients with a COPD diagnosis who attend an annual control visit for COPD.

Main variables: DrCOPD includes information on forced expiratory volume in 1 second, smoking status, body mass index, dyspnea, treatment modalities such as rehabilitation, smoking cessation, medical treatment, and the use of noninvasive ventilation during hospitalization due to exacerbations. The outcome variables include frequency of readmission and death 30 days after discharge from hospital.

Descriptive data: Since 2008, the registration in the Danish hospitals has gradually become more comprehensive. In 2014, $90 \%$ of 16,106 eligible patients had complete data sets that showed an improvement in the processes describing quality of care, including increased offering of smoking cessation, rehabilitation, and correct treatment with inhaled medication. Data from GPs have been included since 2011, but are still incomplete and comprise only $\sim 20 \%$ of all COPD patients seen. The collection of data from GP settings has recently been hampered due to legislative controversies.

Conclusion: In relatively few years, DrCOPD has become increasingly comprehensive and has documented an improvement in the management of COPD in Danish hospitals.

Keywords: chronic obstructive pulmonary disease, quality of care, exacerbations, register

\section{Aim of database}

Chronic obstructive pulmonary disease (COPD) is a common disease in Denmark because of the very high smoking prevalence among both men and women in the past 5 decades. The prevalence of COPD in Denmark is thus, among the highest in the Western world. It has been estimated that $\sim 400,000$ of the total population of $\sim 5.5$ million have obstructive lung function impairment compatible with COPD. ${ }^{1}$ In fact, the risk of death from COPD of a Danish woman is 3.3 times higher than the average in Europe, whereas the corresponding risk for a Danish man is $\sim 1.7$ times higher. ${ }^{2}$ COPD is a considerable burden for the Danish society, both with regard to premature deaths as well as economically due to substantial consumption of health care resources. ${ }^{3,4}$

The Danish register of chronic obstructive pulmonary disease (DrCOPD) is a nationwide database aiming to describe the quality of treatment of all patients with 
diagnosis of COPD in Denmark. The register started in 2008 by including hospital-based data, but in 2011, expanded also to include data from the setting of the general practice (GP). The ultimate goal of DrCOPD is to improve the quality of care for these patients by inspiring health care professionals and by providing data for research.

\section{Study population}

DrCOPD was initiated in 2008 as a part of the Danish National Indicator Project in the hospital setting. ${ }^{5}$ All Danish hospitals treating patients with COPD were included. The register comprises both outpatients with COPD and patients acutely admitted because of an exacerbation of COPD. Each year $\sim 16,000$ outpatients and 21,000 inpatients are included in the register and, in total, $>50,000$ individual patients have been included since 2008 .

The outpatient study population for DrCOPD includes all patients aged 30 years and older who attend any hospital outpatient clinic with the following primary diagnoses according to the Danish version of the International Classification of Diseases, Tenth Revision (ICD-10): COPD (J44.X), or respiratory failure (J96.X) in combination with COPD (J44.X) as a secondary diagnosis.

The inpatients included in DrCOPD were those discharged from hospital with either a primary diagnosis of COPD (J44.X) or a primary diagnosis of respiratory failure (J96.X) or pneumonia (J18.X) together with COPD as a secondary diagnosis. The data were retrieved from the Danish National Patient Registry, which has covered all hospital admissions in Denmark since $1995 .{ }^{6}$ The diagnosis coding was performed by the physicians in charge of the patients at the individual hospital. Table 1 shows the number of individuals included in the hospital setting both as outpatients and as inpatients from 2008 to 2014.

Data from the GPs have been collected since 2011 through the Danish General Practice Database. ${ }^{7}$ This database uses diagnostic coding according to the International Classification of Primary Care (ICPC), which has been implemented in the electronic health record systems in GP in Denmark and, since 2008, has been updated under the name of ICPC2-DK. ${ }^{8}$ The patients with the diagnosis of COPD (R95) according to the ICPC-2-DK are included when they attend an annual checkup. By an agreement since 2009, GPs shall offer this annual checkup to all patients listed, if they fulfill the criteria for COPD. In 2011, data on $\sim 4,000$ patients with COPD were transferred to the DrCOPD. In 2014, this number increased to $\sim 15,000$. Yet, this still constitutes only a minor fraction of the $\sim 100,000-120,000$ patients who are treated with medications for COPD in Denmark. Due to legislative controversies, transmission of all data from GPs was temporarily put to a halt on September 30, 2014, and it is not yet known when and how the data transmission to DrCOPD will be reestablished. The data from GP will not be described further in this brief report. ${ }^{9}$

The validity of the COPD coding practice in the hospital setting has been investigated for both inpatients and outpatients. The latter was investigated by an audit of 20 case records from each of the participating hospitals. This audit comprised 676 outpatients and showed good validity, documenting that the vast majority of outpatients with COPD diagnosis indeed have COPD. The positive predictive value of an outpatient COPD diagnosis was $86 \%$, and $79 \%$ of the patients with a clinical diagnosis of COPD had a spirometric verification of diagnosis within the past 3 years (data published in the 2011 National Audit of COPD). A similar audit of coding practice in connection with acute exacerbations was performed. It included 1,988 records (comprising $\sim 10 \%$ of all acute COPD admissions per year) supplemented with a validation sample of 1,986 cases discharged with the diagnosis of respiratory failure or pneumonia without COPD. This audit showed a positive predictive value of an acute COPD discharge diagnosis of $92 \% .{ }^{10}$ At the same time, this study showed considerable underrecording of COPD in patients with pneumonia and acute respiratory failure, which is in accordance with previous observations. ${ }^{11}$

It has been suggested that data on the quality of municipal care for COPD patients should also be included in

Table I Number of individuals included in Danish Register for COPD in the hospital setting as outpatients and inpatients during the existence of the register

\begin{tabular}{llllllll}
\hline Patients & $\mathbf{2 0 0 8}$ & $\mathbf{2 0 0 9}$ & $\mathbf{2 0 1 0}$ & $\mathbf{2 0 1 1}$ & $\mathbf{2 0 1 2}$ & $\mathbf{2 0 1 3}$ & $\mathbf{2 0 1 4}$ \\
\hline Outpatients $^{\mathrm{a}}$ & 11,730 & 13,153 & 14,841 & 15,264 & 15,649 & 16,098 & 16,106 \\
Inpatients $^{\mathrm{b}}$ & 19,189 & 19,728 & 19,728 & 19,702 & 19,513 & 22,683 & 21,839 \\
\hline
\end{tabular}

Notes: anpatients include patients with main (primary) diagnosis of COPD or patients with primary diagnosis of respiratory failure and secondary diagnosis of COPD (there are no data on the distribution of these two diagnoses); ' Inpatients comprise patients with primary diagnosis of COPD (72\%), patients with primary diagnosis of acute respiratory failure with secondary diagnosis of COPD (II\%), and patients with primary diagnosis of pneumonia with secondary diagnosis of COPD (I7\%). Abbreviation: COPD, chronic obstructive pulmonary disease. 
DrCOPD. The municipalities have an increasingly high responsibility for the care for COPD patients, among others, offering rehabilitation and smoking cessation programs. The steering committee of DrCOPD has recently received participation of representatives from the municipalities, and discussions on indicator development and data collection are in progress.

\section{Main variables}

Main variables recorded in DrCOPD are registered at the outpatient clinic where the patient is followed. Data are continuously uploaded to the Danish National Patient Registry. The same registry provides information on the number of outpatient contacts, hospitalizations, the duration of stay, and mode of in-hospital treatment, including use of invasive and noninvasive ventilation (NIV). The Danish Prescription Registry gives information on medical treatment, and the Danish Civil Registration System gives information on survival. ${ }^{12}$

The variables registered include both indicators describing correct management of COPD (process indicators) and indicators describing the outcomes (result indicators), including 30-day mortality and readmissions after an acute exacerbation. Initially, four evidence-based quality-of-care process indicators were identified for outpatients: lung function assessed as forced expiratory volume in 1 second $\left(\mathrm{FEV}_{1}\right)$ expressed in percentage of predicted value ( $\mathrm{FEV}_{1} \%$ pred), body mass index - assessed as kilograms per square meter, dyspnea assessed using the Medical Research Council (MRC) scale, and smoking status. Additional indicators registered for outpatients were help to stop smoking for smokers and a referral to pulmonary rehabilitation for patients with MRC dyspnea score $>2$. Over the years, the process indicators were expanded to include indicators describing medical treatment (use of long-acting bronchodilators and inhaled steroids) and training in inhalation technique. Table 2 shows the types of variables registered for outpatients from 2008 to 2014 and the indicator fulfillment during this period. Indicator fulfillment is shown as the percentage of cases fulfilling the indicator: eg, if half of all patients had their lung function measured, then the indicator fulfillment is $50 \%$.

The indicators and variables for inpatients include use of NIV during admission, use of invasive ventilation as process indicator, and result indicators describing 30-day mortality and 30-day readmissions. To adjust for comorbidities when comparing mortality and readmissions among hospitals, Charlson comorbidity index has been derived for each inpatient using information from the Danish National
Patient Registry. ${ }^{13}$ Table 3 shows the development from 2008 to 2014 with regard to use of NIV, mortality, and readmissions.

\section{Examples of research}

Each year, DrCOPD produces a standard report in Danish, which is published online at www.Sundhed.dk and focuses on indicator fulfillment at the hospital level, regional level, and in all of Denmark.

To date, data from DrCOPD have been used to publish two papers focusing on the development in quality of care for outpatients with COPD and on the development regarding treatment of acute exacerbation. The first paper documents improved registration of the processes of care defined as high quality of care of COPD. ${ }^{14}$ The second paper focuses on the treatment of COPD patients admitted because of an exacerbation and showed that the use of NIV in Denmark increased from 2008 to 2011, but that regional variation remained and no substantial improvements in mortality were observed. ${ }^{15}$ A number of new studies focusing on changes in smoking habits and medicine adherence among the patients are continuing.

\section{Administrative issues and funding}

Reporting to DrCOPD is mandatory for all Danish hospitals. There is no economic incentive, but there is substantial interest from hospital boards of directors, politicians, and patient organizations regarding both the completeness of registration and the performance of the individual hospitals with regard to indicator fulfillment. Data are collected in accordance with current Danish data protection laws (Sundhedslovens $\S 196$, stk. 4 - law nr 546, dated 24.06.2005/law $\mathrm{nr}$ 603, dated 18.06.2012). Laws and procedures for data access to DrCOPD are governed by the Data Protection Agency (Datatilsynet) and the National Board of Health (Sundhedsstyrelsen). Applications for access to DrCOPD are reviewed by the Chair and Steering Committee following application to The Danish Clinical Registries - a national improvement program (RKKP), which is a nationwide initiative for monitoring and improving the quality of care for many important frequent or chronic diseases or conditions. The funding for the administration of the database and for preparation of the annual reports comes from RKKP.

\section{Conclusion}

Since its initiation in 2008, DrCOPD has enrolled several thousands of individuals with COPD. Through its focus on 


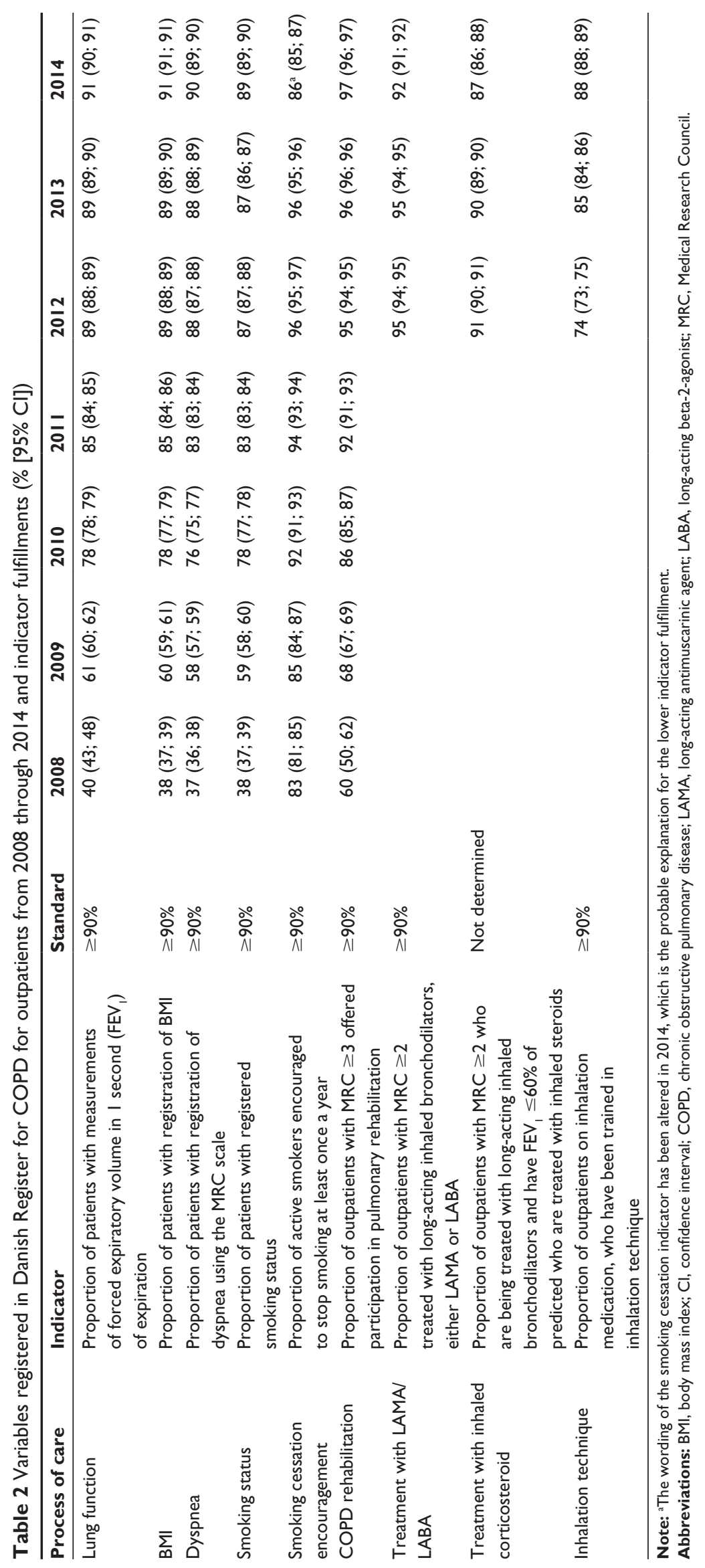


the quality of care, DrCOPD has been a well-functioning instrument for improving the management of care for Danish patients with COPD. In the future, the register will also become a powerful tool for clinical and epidemiological research on COPD.

\section{Acknowledgment}

This paper was funded by the Program for Clinical Research Infrastructure (PROCRIN), established by the Lundbeck Foundation and the Novo Nordisk Foundation, and administered by the Danish Regions.

\section{Disclosure}

The authors report no conflicts of interest in this work.

\section{References}

1. Lange P, Marott JL, Dahl M, Ingebrigtsen TS, Vestbo J, Nordestgaard BG. Substantial need for early diagnosis, rehabilitation and treatment of chronic obstructive pulmonary disease. Dan Med J. 2012;59:A4396.

2. López-Campos JL, Ruiz-Ramos M, Soriano JB. Mortality trends in chronic obstructive pulmonary disease in Europe, 1994-2010: a joinpoint regression analysis. Lancet Respir Med. 2014;2(1): 54-62.

3. Bilde L, Rud SA, Dollerup J, Baekke BH, Lange P. The cost of treating patients with COPD in Denmark - a population study of COPD patients compared with non-COPD controls. Respir Med. 2007;101(3):539-546.

4. Løkke A, Hilberg O, Tønnesen P, Ibsen R, Kjellberg J, Jennum P. Direct and indirect economic and health consequences of COPD in Denmark: a national register-based study: 1998-2010. BMJ Open. 2014;4(1):e004069. doi: 10.1136/bmjopen-2013-004069.

5. Mainz J, Krog BR, Bjørnshave B, Bartels B. Nationwide continuous quality improvement using clinical indicators: the Danish national indicator project. Int J Qual Health Care. 2004;16(suppl 1):i45-i50.

6. Andersen TF, Madsen M, Jørgensen J, Mellemkjær L, Olsen JH. The Danish hospital register. Dan Med Bull. 1999;46(3):263-268.

7. Schroll H, Paulsen MS, Skjoldaa L, Thomsen JL. Danish general practice database. Ugeskr Laeger. 2012;174(42):2541.

8. Rosendal M, Falkø E. Diagnostic classification in Denmark with emphasis on general practice. Ugeskr Laeger. 2009;171(12):997-1000.

9. Jakobsen M, Anker N, Dollerup J, Poulsen PB, Lange P. Study on drug costs associated with COPD prescription medicine in Denmark. Clin Respir J. 2013;7(4):328-337.

10. Thomsen RW, Lange P, Hellqvist B, et al. Predictive values of discharge diagnoses of acute COPD, pneumonia, and respiratory failure for presence of COPD in the Danish national patient registry. Respir Med. 2011;105(7):1063-1068.

11. Jensen HH, Godtfredsen NS, Lange P, Vestbo J. Potential misclassification of causes of death from COPD. Eur Respir J. 2006;28(4): 781-785.

12. Schmidt M, Pedersen L, Sørensen HT. The Danish civil registration system as a tool in epidemiology. Eur J Epidemiol. 2014;29(8):541-549.

13. Charlson ME, Pompei P, Ales KL, MacKenzie CR. A new method of classifying prognostic comorbidity in longitudinal studies: development and validation. J Chronic Dis. 1987;40(5):373-383.

14. Tøttenborg SS, Thomsen RW, Nielsen H, Johnsen SP, Frausing Hansen E, Lange P. Improving quality of care among COPD outpatients in Denmark 2008-2011. Clin Respir J. 2013;7(4):319.

15. Tøttenborg SS, Johnsen SP, Thomsen RW, Nielsen H, Hansen EF, Lange P. Use of non-invasive ventilation is increasing in patients admitted with a chronic obstructive pulmonary disease exacerbation. Dan Med J. 2013;60:A4686. 


\section{Publish your work in this journal}

Clinical Epidemiology is an international, peer-reviewed, open access, online journal focusing on disease and drug epidemiology, identification of risk factors and screening procedures to develop optimal preventative initiatives and programs. Specific topics include: diagnosis, prognosis, treatment, screening, prevention, risk factor modification,

Submit your manuscript here: http://www.dovepress.com/clinical-epidemiology-journal systematic reviews, risk \& safety of medical interventions, epidemiology \& biostatistical methods, and evaluation of guidelines, translational medicine, health policies \& economic evaluations. The manuscript management system is completely online and includes a very quick and fair peer-review system, which is all easy to use. 